\title{
The effect of clove extract on the minimum inhibitory concentration of ciprofloxacin in fluoroquinolone resistant clinical isolates of Staphylococcus aureus
}

\author{
Nouran Hamed Assar ${ }^{1}$, Yasser Musa Ibrahim ${ }^{1}$, Ahmed Megahed Abouwarda ${ }^{2 \star}$ and \\ Magdy Ali Amin ${ }^{3}$
}
${ }^{1}$ General Department of Basic Medical Sciences, Microbiology Department, National Organization for Drug Control and Research (NODCAR), Giza, Egypt.
${ }^{2}$ Biology Department, Faculty of Science and Arts-Khulais, King Abdulaziz University, Saudi Arabia.
${ }^{3}$ Microbiology and Immunology Department, Faculty of Pharmacy, Cairo University, Giza, Egypt.

Accepted 27 January, 2012

\begin{abstract}
Multidrug resistant strains of Staphylococcus aureus cause serious problems worldwide. We have characterized the quinolone resistant mechanisms in clinical isolates of $S$. aureus and tested their susceptibilities to other classes of antibiotics. Results of this study showed high level of resistance to quinolone in the selected isolates and suggested that resistance is due to mutations in gyrA and parC genes in addition to efflux mechanism. The selected strains were not only resistant to quinolones but also found resistant to a number of other antibiotics. This study also shed some light on the possibility of using Clove as an efflux pump inhibitor. The minimum inhibitory concentration of ciprofloxacin against multidrug resistant isolates was reduced from 256 to $64 \mu \mathrm{g} / \mathrm{ml}$ when used in combination with Clove extract. This result indicated that Clove is a potential efflux pump inhibitor. In addition, the effect of different efflux pump inhibitors on the resistance of these isolates was determined.
\end{abstract}

Key words: Staphylococcus aureus, fluoroquinolone antibiotics, ciprofloxacin, efflux pump inhibitor, clove.

\section{INTRODUCTION}

Staphylococcus aureus is a Gram positive human pathogen that causes a wide range of infections, some of which can be life-threatening such as septicemia, pneumonia, osteomyelitis and endocarditis (Pan et al., 2002). This organism is responsible for significant morbidity and mortality in intensive care units worldwide. Fluoroquinolones are broad-spectrum antibacterial agents that act on bacteria by inhibiting their DNA synthesis. They are known to have two enzyme targets, DNA gyrase and topoisomerase IV, in the bacterial cell. Both enzymes are essential for bacterial DNA replication (Drlica and Zhao, 1997).

Antibiotic resistance is a serious problem throughout

${ }^{*}$ Corresponding author. E-mail: ahmedabouwarda@gmail.com. Tel: 00966560011481. the world. Bacterial pathogens resistant to multiple antibiotics continue to emerge at a higher rate. Resistance to fluoroquinolones in bacteria may result from point mutations within DNA gyrase (encoded by gyrA and gyrB genes). This causes a reduction in the affinity of the enzyme for fluoroquinolones and thereby decreases the susceptibility of the organisms to these antibiotics (Everett et al., 1996; Ruiz, 2003). Mutations in parC and parE genes which encode for topoisomerase IV can also cause fluoroquinolone resistance (Ruiz, 2003). In $S$. aureus, several studies have shown that a combination of mutations in genes for both enzymes can cause high-level of fluoroquinolone resistance (Hooper, 2002; Horii et al., 2003; lihara et al., 2006; Wang et al., 1998).

Another mechanism that can lead to fluoroquinolone resistance is active efflux by drug transporters such as NorA, NorB, and NorC (DeMarco et al., 2007; Ding et al., 
Table 1. List of oligonucleotide primers used in this study.

\begin{tabular}{llcl}
\hline Primer name & Primer sequence $\left(5^{\prime} \mathbf{~}^{\prime}\right.$ ) & Product size (base pair) & Reference \\
\hline $\begin{array}{l}\text { parC } \mathrm{F} \\
\text { parC } \mathrm{R}\end{array}$ & $\begin{array}{l}\text { GTAGGTGGCAAGCGTTATCC } \\
\text { CGCACATCAGCGTCAG }\end{array}$ & 458 & (Trong et al., 2005) \\
$\begin{array}{l}\text { gyrA } \mathrm{F} \\
\text { gyrA } \mathrm{R}\end{array}$ & $\begin{array}{l}\text { TATTACCAGTGAAATGCGTGAATC } \\
\text { ACGAGAACGCATTTGAATTGAACC }\end{array}$ & 700 & (Discotto et al., 2001) \\
$\begin{array}{l}\text { norA } \mathrm{F} \\
\text { norA } \mathrm{R}\end{array}$ & $\begin{array}{l}\text { ATGTTTGCAGTTGGCCACAATTTTTC } \\
\text { ATGACCAAGCTATAAATGTTAAC }\end{array}$ & \multirow{2}{*}{575} & \\
\hline
\end{tabular}

F: forward; R: reverse.

2008; Truong-Bolduc et al., 2006). Efflux pump genes and proteins are present in both antibiotic-susceptible and antibiotic-resistant bacteria. Antimicrobial resistance is due to either increased expression of efflux pump protein or amino acid substitutions of the efflux protein that make the export process more efficient. Efflux pumps may also be involved in multidrug resistance (MDR) by transporting a range of different antimicrobial classes. Overexpression of NorB in $S$. aureus was associated with decreased susceptibility to fluoroquinolone, tetracycline, disinfectants and dyes (Truong-Bolduc et al., 2005).

One of the approaches towards combating drug resistance is to use natural or synthetic compounds in combination with the antibiotic in use to restore the activity of the drug (Sabatini et al., 2008). Therefore, development of what is known as efflux pump inhibitors (EPIs) that decrease the effectiveness of drug transporters would be an advance to provide successful treatment of multidrug resistant organisms. Such inhibitors will increase antibacterial effectiveness, expand the spectrum of antibacterial activity, reverse resistance and reduce the rates of resistance development. Piperine is one of these compounds which was found to inhibit NorA efflux pump in $S$. aureus and decrease the minimum inhibitory concentration of ciprofloxacin resistant strains (Khan et al., 2006).

The presented study characterizes fluoroquinolone resistance mechanisms in clinical isolates of $S$. aureus and determines the effect of different efflux pump inhibitors on the resistance of these isolates.

\section{MATERIALS AND METHODS}

\section{Bacterial isolates, culture media and growth conditions}

One hundred clinical isolates of suspected $S$. aureus were collected from El-Demerdash hospital. S. aureus ATCC 29737, which is sensitive to ciprofloxacin, was used as a control strain and was taken the symbol "STAPH S" in this study. All isolates were routinely grown on nutrient agar medium (Oxoid) by incubation at $37^{\circ} \mathrm{C}$ for $24 \mathrm{~h}$ and stored at $-70^{\circ} \mathrm{C}$. Other culture media including DNAse agar, Mannitol salts agar and broth, Muller Hinton agar and broth, Paired-Barker agar, Tryptic soya agar and broth were obtained from Oxoid and were employed as necessary. Isolates were confirmed as $S$. aureus by examining Gram-stained cultures, growth on specific media and by testing their ability or inability to produce specific enzymes such as catalase, coagulase, DNAse and oxidase.

\section{Antibiotic susceptibility testing}

Antibiotic discs were purchased from Oxoid Company. 6-mm disc impregnated with Chloramphenicol, Cefaclor, Cefadroxil, Ciprofloxacin, Erythromycin, Levofloxacin, Norfloxacin, Sulphonamides and Trimethoprime, and Tobramycin were used according to the manufacturers' recommendations. The susceptibility of tested isolates to antibiotics was determined by disc diffusion method according to CLSI M100-S21 guidelines (2011). Antibiotic discs were applied onto Muller Hinton agar plates seeded with the tested organism and incubated at $37^{\circ} \mathrm{C}$ for $24 \mathrm{~h}$. After incubation, the diameters of inhibition zones were measured and isolates were classified as susceptible, intermediate or resistant according to the guidelines of CLSI M100-S21 (2011). The minimum inhibitory concentrations (MICs) of ciprofloxacin were determined using agar dilution method in Muller Hinton agar plates according to a standard protocol (EUCAST, 2000). Ciprofloxacin was dissolved in distilled $\mathrm{H}_{2} \mathrm{O}$ and diluted to give serial two-fold dilutions ranging from 512 to $4 \mu \mathrm{g} / \mathrm{ml}$. Before gelling, $20 \mathrm{ml}$ of agar medium were added to each of the Petri dishes containing the antibiotic and the Petri dishes were swirled carefully until the agar began to set. Subsequently, $2 \mu$ l of each bacterial strain $\left(10^{4} \mathrm{cfu} / \mathrm{ml}\right)$ were inoculated on the Mueller Hinton agar surface. MIC was defined as the lowest antibiotic concentration, showing no visible bacterial growth after incubation time ( $37^{\circ} \mathrm{C}$ for $\left.24 \mathrm{~h}\right)$.

\section{Amplification of quinolone resistance determining region (QRDR), gyrA and parC and the drug transporter norA by PCR}

Chromosomal DNA was prepared from selected isolates as described in a published procedure (Sambrook et al., 1989). The gyrA, parC and norA gene fragments were amplified using primers shown in Table 1. The PCR conditions were set as described by Jonas et al. (2002).

\section{Sequence analysis of gyrA and parC genes}

The PCR products were purified with the DNA purifying kit (Qiagen) according to the manufacture's instructions. The samples were processed with DNA sequencing kit and analyzed in an automatic DNA sequencer (Shi et al., 2005). The sequence was aligned with 
the software Finch TV and the sequence homology searches were performed using BLAST program (www.ncbi.nlm.nih.gov/blast).

\section{Quinolone accumulation assay}

Ciprofloxacin uptake was assayed using a fluorometric method. Cells were grown to an $A_{660}$ of 0.4 , harvested by centrifugation,

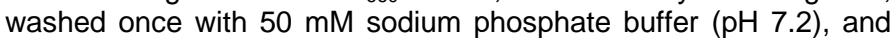
suspended in the same buffer to $20 \mathrm{~A}_{660}$ units per $\mathrm{ml}$ (approximately $40 \mathrm{mg}$ [wet weight] per $\mathrm{ml}$ ). Ciprofloxacin was added to a final concentration of $10 \mu \mathrm{g} / \mathrm{ml}$, and at appropriate time intervals. $0.5-\mathrm{ml}$ samples were removed and diluted into $2.0 \mathrm{ml}$ of phosphate buffer. The cells were pelleted $(5,600 \times \mathrm{g}$ for $1 \mathrm{~min})$, washed once with 2.0 $\mathrm{ml}$ of buffer, and treated with $2.0 \mathrm{ml}$ of $0.1 \mathrm{M}$ glycine hydrochloride $(\mathrm{pH} 3.0)$ for $1 \mathrm{~h}$. The samples were centrifuged (5,600 $\mathrm{xg}$ for $5 \mathrm{~min})$, and the fluorescence of the supernatant was determined at $442 \mathrm{~nm}$ with excitation at $282 \mathrm{~nm}$ using a Spectroflurometer (Jonas et al., 2002).

\section{Purification of piperine and preparation of clove extract}

Piperine was purified from black pepper according to a published protocol (Epstein et al., 1993). Hundred grams of dried cloves (Syzygium aromaticum) were extracted in $400 \mathrm{ml}$ of Diethyl ether. The solvent was then removed under reduced pressure in a rotary evaporator (EYELA). Extract was first filtered using Whatman No. 2 filter paper; filtrate was evaporated to dryness at RT in a steady air current. The crude extract was stored at $-20^{\circ} \mathrm{C}$ until required for testing. The extract was dissolved in $10 \%$ dimethyl sulfoxide (DMSO) before use.

\section{In vitro evaluation of efflux pump inhibitors (EPIs)}

Carbonyl cyanide-m chlorophenyl hydrazone (CCCP), omeprazole, piperine (purified from black pepper), reserpine, and clove (diethyl ether extract) were evaluated as EPIs on the quinolone resistant $S$. aureus isolates. The EPIs were tested in combination with ciprofloxacin by a checkerboard titration assay in plates (Vidaillac et al., 2007). The bacterial inoculum was prepared as described above for MIC determinations. Ciprofloxacin and EPIs were tested at eight concentrations $(512$ to $4 \mu \mathrm{g} / \mathrm{ml})$. The Ciprofloxacin or the EPIs alone at the same concentrations were also included. The plates were incubated at $37^{\circ} \mathrm{C}$ for $18 \mathrm{~h}$. The plates were read visually after incubation at $37^{\circ} \mathrm{C}$ for $18 \mathrm{~h}$.

\section{RESULTS}

\section{Identification of isolates}

All clinical isolates were identified at the genus level by examining Gram-stained cultures and growth on specific media. All isolates appeared as grape like cocci characteristic to Staphylococci. All isolates gave the characteristic shape of colonies on Mannitol salts agar medium, Baird-Parker agar medium, and Vogel-Johnson agar medium (data not shown). Among the 100 collected isolates, 46 isolates were found to be catalase positive, coagulase positive, DNAse positive and oxidase negative (data not shown) and therefore confirmed as Staphylococcus aureus.

\section{Susceptibility of isolates to fluroquinolone}

The susceptibility of all $S$. aureus isolates were tested against fluoroquinolone antibiotics (Cibrofloxacin, Norfloxacin and Levofloxacin) in a concentration of 5 $\mu \mathrm{g} / \mathrm{ml}$. Four of these isolates were resistant to the three tested fluoroquinolone antibiotics (data not shown). The MIC of ciprofloxacin against all four resistant isolates was $256 \mu \mathrm{g} / \mathrm{ml}$. These four isolates were selected for further investigations.

\section{Antibiogram of the selected $S$. aureus isolates}

The four fluoroquinolone resistant $S$. aureus isolates (designated as STAPH N3, N5, N26 and N83) were tested for their susceptibility to different classes of antibiotics by using disk diffusion method. A sensitive control S. aureus ATCC 29737 strain (STAPH S) was also included in this test. The antibiotic resistance profiles of the isolates against six antimicrobial agents are presented in Table 2. The results indicate that the majority of isolates were resistant to all antibiotics tested.

\section{Determination of both QRDR ( $g y r A$ and parC) and the drug transporter norA by PCR}

Molecular detections of $S$. aureus gyrA, parC and norA genes using PCR revealed an amplified product in all tested samples and the sizes of the amplicons obtained from the tested strains corresponded to the predicted sizes (data not shown). Sequence analysis of the gyrA and parC genes demonstrated a number of point mutations at different parts of the sequenced genes (data not shown).

\section{Quinolone uptake}

The Ciprofloxacin uptakes of the sensitive control strain of $S$. aureus ATCC 29737 and that of the resistant isolate STAPH N5 (a representative of the quinolone resistant isolates) were measured by fluorometric assay. Figure 1 shows the ciprofloxacin accumulation in both strains. The presented data indicate a decrease in the uptake of ciprofloxacin in the resistant isolate compared to that of the sensitive strain.

\section{Effect of efflux pump inhibitors on ciprofloxacin MIC against resistant isolates}

The possible reduction of ciprofloxacin MIC (originally256 $\mu \mathrm{g} / \mathrm{ml}$ ) in the four resistant isolates when using a combination of ether extract of clove and ciprofloxacin was tested compared with known efflux pump inhibitors. Table 3 shows the effect of these combinations on the 
Table 2. Susceptibility of $S$. aureus isolates to different classes of antibiotics.

\begin{tabular}{lccccc}
\hline \multirow{2}{*}{ Antibiotic } & \multicolumn{5}{c}{ Susceptibility profile of S. aureus isolates } \\
\cline { 2 - 6 } & STAPH N3 & STAPH N5 & STAPH N26 & STAPH N83 & STAPH S \\
\hline Chloroamphenicol & $\mathrm{I}$ & $\mathrm{R}$ & $\mathrm{R}$ & $\mathrm{R}$ & $\mathrm{R}$ \\
Tobramycin & $\mathrm{R}$ & $\mathrm{R}$ & $\mathrm{R}$ & $\mathrm{R}$ & $\mathrm{S}$ \\
Cefaclor & $\mathrm{R}$ & $\mathrm{R}$ & $\mathrm{R}$ & $\mathrm{R}$ & $\mathrm{S}$ \\
Cefadroxil & $\mathrm{R}$ & $\mathrm{R}$ & $\mathrm{R}$ & $\mathrm{R}$ & $\mathrm{S}$ \\
Sulphonamides and Trimethoprime & $\mathrm{S}$ & $\mathrm{R}$ & $\mathrm{R}$ & $\mathrm{S}$ & $\mathrm{S}$ \\
Erythromycin & $\mathrm{R}$ & $\mathrm{R}$ & $\mathrm{R}$ & $\mathrm{R}$ & $\mathrm{S}$ \\
\hline
\end{tabular}

R: resistant; S: sensitive; I: intermediate; STAPH S: S. aureus ATCC 29737 (sensitive control).

Table 3. MIC $(\mu \mathrm{g} / \mathrm{ml})$ of Ciprofloxacin in combination with Piperine, Omeprazole, Reserpine, CCCP and diethyl ether clove extract against 4 clinical isolates of $\mathrm{S}$. aureus using agar dilution method.

\begin{tabular}{lcccc}
\hline Combination & \multicolumn{4}{c}{ S. aureus isolate number } \\
\cline { 2 - 5 }$(\mu \mathrm{g} / \mathrm{ml}) /(\mu \mathrm{g} / \mathrm{ml})$ & STAPH N3 $(\boldsymbol{\mu g})$ & STAPH N5 $(\boldsymbol{\mu g})$ & STAPH N26 $(\boldsymbol{\mu g})$ & STAPH N83 $(\boldsymbol{\mu g})$ \\
\hline Ciprofloxacin/Piperine & $64 / 64$ & $256 / 4$ & $64 / 512$ & $64 / 256$ \\
Ciprofloxacin/Omeprazole & $128 / 128$ & $128 / 128$ & $128 / 8$ & $128 / 64$ \\
Ciprofloxacin/Reserpine & $128 / 4$ & $128 / 4$ & $64 / 128$ & $128 / 4$ \\
Ciprofloxacin/CCCP & $8 / 32$ & $128 / 16$ & $32 / 32$ & $32 / 32$ \\
Ciprofloxacin/Clove extract & $64 / 16$ & $64 / 16$ & $64 / 16$ & $64 / 16$ \\
\hline
\end{tabular}

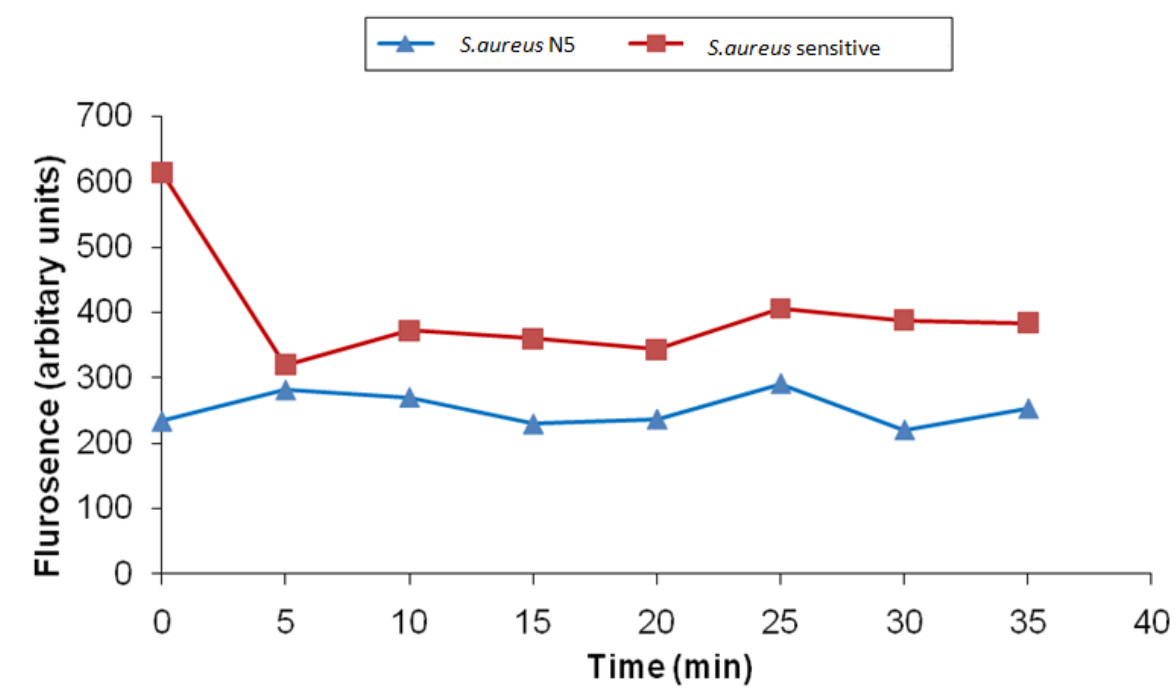

Figure 1. Ciprofloxacin accumulation assay of S. aureus ATCC 29737 and the resistant isolate STAPH N5.

ciprofloxacin MICs. The tested EPIs and Clove extract were used at concentrations below their MICs. The results show that there was a twofold reduction in the MIC of ciprofloxacin in combination with omeprazole and reserpine, and fourfold reduction with piperine and diethyl ether extract of clove. In case of the combination of ciprofloxacin and CCCP, the reduction was ranged from twofold to eightfold.

\section{DISCUSSION}

Development of the fluoroquinolone class of antimicrobial agents advanced the possibility of an effective option for the therapy of serious infections caused by multi-resistant strains of $S$. aureus, even by the oral route of administration. Unfortunately, shortly after introduction of these agents into clinical use, the emergence of 
fluoroquinolone-resistant $S$. aureus was noted (Peterson et al., 1990).

The primary targets of fluoroquinolones are two essential enzymes of bacterial cells, DNA gyrase and topoisomerase IV (Hooper, 2002; Horii et al., 2003; Wang et al., 1998). In most bacterial species the mutations in the genes that lead to fluoroquinolone resistance are limited to a few point mutations at restricted positions of the genes called QRDR. Fluoroquinolone resistance in $S$. aureus is generally associated with two single-point mutations in gyrA at codon 84, and in parC at codon 80 or 84. S. aureus isolates with higher levels of resistance are associated with the second mutation in parC at codon 80 or 84 , depending on the position of the first mutation. When the second mutation in gyrA occurs at codon 85 or 88 , in addition to the first mutation at codon 84 , the strain shows the highest fluoroquinolone resistance even to newer fluoroquinolones (lihara et al., 2006).

In this study, clinical isolates of $S$. aureus were resistant to the three tested fluoroquinolones. The MIC of ciprofloxacin against these isolates was $256 \mu \mathrm{g} / \mathrm{ml}$. The same level of resistance was observed in Escherichia coli in previous studies (Goldstein and Acar, 1995; Murphy et al., 1997).

This high level of resistance could be attributed to mutations in both gyrA and parC which was reported to raise resistance in $S$. aureus (Decousser et al., 2002). The majority of these isolates were also resistant to six other classes of antibiotics. We characterized the mechanism of resistance in these isolates by sequencing of gyrA and parC. To elucidate whether an efflux system was responsible for the multidrug resistance in the tested isolates, accumulation experiment was performed with ciprofloxacin.

Decreased quinolone accumulation is associated with an increase in bacterial permeability to antibacterial agents or the overexpression of efflux pumps (Everett et al., 1996; Ruiz, 2003). Results of this study showed a decreased accumulation of ciprofloxacin in the tested isolate compared with a sensitive control strain. This suggests that the efflux mechanism is present and active in the tested isolate.

The MIC of the Clove extract was determined in order to use it at a concentration that has no antibacterial activity, a condition of any compound to be used as a safe EPI. The ether extract of Clove was used in combination with the antibiotic ciprofloxacin and bioevaluated against chosen $S$. aureus isolates. Other four known efflux pump inhibitors namely piperine, omeprazole, reserpine and CCCP were also used for the comparative studies. The potentiation of activity of ciprofloxacin in the presence of EPIs was reflected in the reduced MIC of combination compared to that of ciprofloxacin alone. Four-fold reduction in ciprofloxacin MIC was observed when combined with Clove extract, a result indicating that Clove could be a potential EPI.

Previous work on pump inhibitors in $S$. aureus includes the screening of a synthetic library against the NorA transporter (Markham et al., 1999). These inhibitors acted in a synergic manner with ciprofloxacin and significantly suppressed the emergence of ciprofloxacin-resistant $S$. aureus. Beside that, a number of MDR pump inhibitors against the human pathogen $S$. aureus have been described by the Lewis group. Berberine, isolated from Berberis fremontii, is an alkaloid with only weak antibacterial activity ( $\mathrm{MIC}=256 \mathrm{mg} / \mathrm{L}$ ) against a wild-type strain of S. aureus (Stermitz et al., 2000). However, the isolation of the flavonolignan 50-methoxyhydnocarpin-D (5'-MHC-D) and a synergistic study on these two compounds led to a 16 -fold increase in the antibacterial activity of berberine (MIC $=16 \mathrm{mg} / \mathrm{L}$ ) (Stermitz et al., 2000). 5'-MHC-D also had a synergistic effect with several other NorA substrates, including norfloxacin.

In addition, ciprofloxacin was incorporated at subinhibitory concentrations $(1 / 8 \mathrm{MIC})$ to the crude chloroform extracts of Jatropha elliptica and the mixture assayed against NorA expressing $S$. aureus, the activity of the extract was enhanced. This suggests the presence of an inhibitor of the pump which could restore the activity of Ciprofloxacin (Marquez et al., 2005).

In conclusion, the results showed in this study highlight the effect of efflux pump inhibitors on the resistance of $S$. aureus and suggest a potential role of Clove as an efflux pump inhibitor.

\section{REFERENCES}

Clinical and Laboratory Standards Institute (2011). Performance standards for antimicrobial susceptibility testing: nineteen informational supplement (CLSI M100-S21), Wayne, PA: CLSI.

Decousser JW, Allouch PY, Courvalin P Leclercq R (2002). In vitro activity of moxifloxacin against recent community-acquired respiratory tract pathogens isolated in France: a national survey. Int. J. Antimicrob. Agents, 20: 186-195.

DeMarco CE, Cushing LA, Frempong-Manso E, Seo SM, Jaravaza TA, Kaatz GW (2007). Efflux-related resistance to norfloxacin, dyes, and biocides in bloodstream isolates of Staphylococcus aureus. Antimicrob. Agents Chemother., 51: 3235-3239.

Ding Y, Onodera Y, Lee JC, Hooper DC (2008). NorB, an efflux pump in Staphylococcus aureus strain MW2, contributes to bacterial fitness in abscesses. J. Bacteriol., 190: 7123-7129.

Discotto LF, Lawrence LE, Denbleyker KL, Barrett JF (2001). Staphylococcus aureus mutants selected by BMS-284756. Antimicrob. Agents Chemother., 45: 3273-3275.

Drlica K, Zhao X (1997). DNA gyrase, topoisomerase IV, and the 4quinolones. Microbiol. Mol. Biol. Rev., 61: 377-392.

Epstein WW, Netz DF, Seidel JL (1993). Isolation of piperine from black pepper. J. Chem. Educ., 70: 598-599.

EUCAST (2000). EUCAST Definitive Document E.DEF 3.1, June 2000: Determination of minimum inhibitory concentrations (MICs) of antibacterial agents by agar dilution. Clin. Microbiol. Infect., 6: 509515.

Everett MJ, Jin YF, Ricci V, Piddock LJ. (1996). Contributions of individual mechanisms to fluoroquinolone resistance in 36 Escherichia coli strains isolated from humans and animals. Antimicrob. Agents Chemother., 40: 2380-2386.

Goldstein FW, Acar JF (1995). Epidemiology of quinolone resistance. Europe and North and South America. Drugs 49 Suppl., 2: 36-42.

Hooper DC (2002). Fluoroquinolone resistance among Gram-positive cocci. Lancet. Infect. Dis., 2: 530-538.

Horii T, Suzuki Y, Monji A, Morita M, Muramatsu H, Kondo Y, Doi M, Takeshita A, Kanno T, Maekawa M (2003). Detection of mutations in 
quinolone resistance-determining regions in levofloxacin- and methicillin-resistant Staphylococcus aureus: effects of the mutations on fluoroquinolone MICs. Diagn. Microbiol. Infect. Dis., 46: 139-145.

lihara $H$, Suzuki T, Kawamura Y, Ohkusu K, Inoue Y, Zhang W, Monir Shah M, Katagiri Y, Ohashi Y, Ezaki T (2006). Emerging multiple mutations and high-level fluoroquinolone resistance in methicillinresistant Staphylococcus aureus isolated from ocular infections. Diagn. Microbiol. Infect. Dis., 56: 297-303.

Jonas D, Speck M, Daschner FD, Grundmann H (2002). Rapid PCRbased identification of methicillin-resistant Staphylococcus aureus from screening swabs. J. Clin. Microbiol., 40: 1821-1823.

Khan IA, Mirza ZM, Kumar A, Verma V, Qazi GN (2006). Piperine, a phytochemical potentiator of ciprofloxacin against Staphylococcus aureus. Antimicrob. Agents Chemother., 50: 810-812.

Markham PN, Westhaus E, Klyachko K, Johnson ME, Neyfakh AA. (1999). Multiple novel inhibitors of the NorA multidrug transporter of Staphylococcus aureus. Antimicrob. Agents Chemother., 43: 24042408.

Marquez B, Neuville L, Moreau NJ, Genet JP, dos Santos AF, Cano de Andrade MC, Sant'Ana AE (2005). Multidrug resistance reversal agent from Jatropha elliptica. Phytochemistry, 66: 1804-1811.

Murphy OM, Marshall C, Stewart D, Freeman R (1997). Ciprofloxacinresistant Enterobacteriaceae. Lancet, 349: 1028-1029.

Pan XS, Hamlyn PJ, Talens-Visconti R, Alovero FL, Manzo RH, Fisher LM (2002). Small-colony mutants of Staphylococcus aureus allow selection of gyrase-mediated resistance to dual-target fluoroquinolones. Antimicrob. Agents Chemother., 46: 2498-2506.

Peterson LR, Quick JN, Jensen B, Homann S, Johnson S, Tenquist J, Shanholtzer C, Petzel RA, Sinn L, Gerding DN (1990). Emergence of ciprofloxacin resistance in nosocomial methicillin-resistant Staphylococcus aureus isolates. Resistance during ciprofloxacin plus rifampin therapy for methicillin-resistant $S$ aureus colonization. Arch. Int. Med., 150: 2151-2155.

Ruiz J (2003). Mechanisms of resistance to quinolones: target alterations, decreased accumulation and DNA gyrase protection. J. Antimicrob. Chemother., 51: 1109-1117.

Sabatini S, Kaatz GW, Rossolini GM, Brandini D, Fravolini A. (2008). From phenothiazine to 3-phenyl-1,4-benzothiazine derivatives as inhibitors of the Staphylococcus aureus NorA multidrug efflux pump. J. Med. Chem., 51: 4321-4330.
Sambrook J, Fritsch EF, Maniatis T (1989). Molecular cloning: a laboratory manual. Cold Spring Harbor, NY: Cold Spring Harbor Laboratory Press.

Shi WF, Jiang JP, Xu N, Huang ZM, Wang YY (2005). Inhibitory effects of reserpine and carbonyl cyanide m-chloro-phenylhydrazone on fluoroquinolone resistance of Acinetobacter baumannii. Chin. Med. J. Engl., 118: 340-343.

Stermitz FR, Lorenz P, Tawara JN, Zenewicz LA, Lewis K (2000). Synergy in a medicinal plant: antimicrobial action of berberine potentiated by 5 '-methoxyhydnocarpin, a multidrug pump inhibitor. Proc. Natl. Acad. Sci. USA, 97: 1433-1437.

Truong HN, Prunier AL, Leclercq R (2005). Hypermutable and fluoroquinolone-resistant clinical isolates of Staphylococcus aureus. Antimicrob. Agents Chemother., 49: 2098-2101.

Truong-Bolduc QC, Dunman PM, Strahilevitz J, Projan SJ, Hooper DC (2005). MgrA is a multiple regulator of two new efflux pumps in Staphylococcus aureus. J. Bacteriol., 187: 2395-2405.

Truong-Bolduc QC, Strahilevitz J, Hooper DC (2006). NorC, a new efflux pump regulated by MgrA of Staphylococcus aureus. Antimicrob. Agents Chemother., 50: 1104-1107.

Vidaillac C, Guillon J, Arpin C, Forfar-Bares I, Ba BB, Grellet J, Moreau S, Caignard DH, Jarry C, Quentin C (2007). Synthesis of omeprazole analogues and evaluation of these as potential inhibitors of the multidrug efflux pump NorA of Staphylococcus aureus. Antimicrob. Agents Chemother., 51: 831-838.

Wang T, Tanaka M, Sato K (1998). Detection of grlA and gyrA mutations in 344 Staphylococcus aureus strains. Antimicrob. Agents Chemother., 42: 236-240.

Yamada H, Kurose-Hamada S, Fukuda Y, Mitsuyama J, Takahata M, Minami S, Watanabe Y, Narita H (1997). Quinolone susceptibility of norA-disrupted Staphylococcus aureus. Antimicrob. Agents Chemother., 41: 2308-2309. 